\title{
Day of Launch Profile Selection for Pad Abort Guidance
}

\author{
Ryan J. Whitley ${ }^{1}$ and Ryan W. Proud ${ }^{2}$ \\ NASA Johnson Space Center, Houston, TX, 77058
}

\begin{abstract}
A day of launch selection approach that involves choosing from an array of pitch profiles of varying loft was analyzed with the purpose of reducing the risk of a land landing failure during a pad abort. It was determined that selecting from three pitch profiles based on winds of the day can reduce the probability of a land landing significantly without compromising other critical performance metrics.
\end{abstract}

\section{Introduction}

$\mathrm{T}$

HE Orion pad abort GN\&C system is designed to "place the LAV (Launch Abort Vehicle) on a trajectory that provides the shortest distance to the shoreline from the launch pad in order to achieve a water landing". ${ }^{1}$ The rationale behind this trajectory profile is that the nominal vehicle structure is only designed to withstand a water landing, so the inability to achieve this condition is considered an abort failure. However, a pitch profile cannot be designed solely to reach the water; if the angle of attack is too large during the abort motor firing, the depressed trajectory and reduced timeline can increase the risk of a main chute inflation failure. A main chute failure is more objectionable than a land landing due to its higher impact on overall loss-of-crew (LOC) probabilities. Because of this tradeoff, a significant driver in ensuring a successful pad abort relates directly to the particular wind of the day.

It is this fact that prompts the design of wind-tailored pitch maneuver profiles. In general, there appears to be two types of wind days for a launch from KSC: those that provide tailwinds and aid in pushing the vehicle towards the water and those that provide headwinds and impede this motion. This paper introduces a guidance technique that tailors the pad abort pitch profile to these two types of winds. The idea is to reduce the size of or replace the need for wind placards that would decrease launch availability, and the degree to which this is effective will justify the use of this type of I-load.

\section{Methodology}

\section{A. Pitch Profile Design}

The pitch profiles were designed based off two categories of winds: tailwinds, or those generally favorable for a water landing and headwinds, or those generally unfavorable for a water landing. For each category a representative wind was selected so that the pitch profile tables were the only independent variables. These tables were then varied to produce varying degrees of angle of attack in order to generate a curve for each wind as shown in the cartoon in Figure 1. For example, pitch profiles A1-A4 are designed for the tailwind while profiles B1-B4 are designed for the headwind. Based on their respective performance, the profiles are then selected for further analysis.

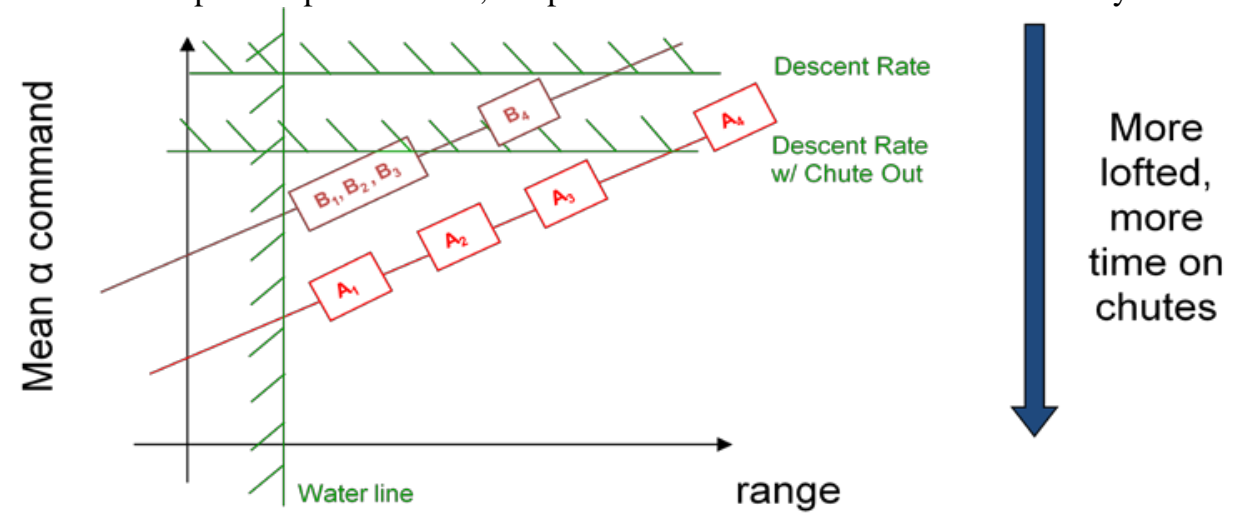

Figure 1. Methodology for establishing pitch profiles based on head and tailwinds

\footnotetext{
${ }^{1}$ Aerospace Engineer, Orion GN\&C, NASA Johnson Space Center, AIAA Member

${ }^{2}$ Ascent Abort Mode Team Lead, Orion GN\&C, NASA Johnson Space Center, AIAA Member
}

1

American Institute of Aeronautics and Astronautics 
The green constraint lines shown in Figure 1 outline the limits for a successful pad abort due to either falling short of the waterline (where the angle of attack command is smaller) or descent rate failure (where the angle of attack is larger). The second horizontal line is a tighter constraint that protects for a chute failure (drogue or main). It is expected that a higher average angle of attack during abort motor firing will succeed in deploying chutes further out to sea but also reduce flight time which explains the positive slope of the profile curves.

Once these curves were drawn with real data, pitch profiles were selected for individual pad abort Monte Carlos that measured the impact of real dispersions. Based on an analysis of the resulting failures a categorization process was set up to select profiles for a given day in a number of different scenarios based on wind magnitude. The way in which this categorization is completed served as the basis for comparison for the trade study. The amount of performance margin on the green constraint lines for a given profile to produce the desired results for the Monte Carlo was unknown at the beginning of the analysis, so iteration on the profile design was required. In addition to the descent rate constraints shown in Fig. 1 the vehicle can also lose control during the firing of the abort motor due to an overly aggressive profile. This failure, referred to as tumbling, is even more undesirable than violating the touchdown velocity (descent rate) constraint.

\section{B. Chute Architecture Dependence}

The results compiled in this paper are developed in the midst of an ever evolving Orion LAV design. The original pad abort architecture included a drogue to main chute sequence. After a significant redesign of the vehicle chutes, the sequence for pad and low altitude aborts was switched to go straight to mains and skip the drogue stage. In addition, the dynamic pressure limit for the main chutes at line stretch was increased. On the GN\&C side, the LAV control algorithm was modified to accommodate the chute change including the pitch profile control parameters. As a result of these changes, the strategy for pitch profile shifted. The modified LAV control algorithm increased pitch profile design flexibility with a new parameterization of the control variables. But while the trade space appeared to widen with increased performance margin on the dynamic pressure constraint limit, the tumbling and touchdown velocity constraints remain fixed and continue to drive the design.

\section{Drogue to Main Chute Architecture Results}

\section{A. Wind Based Pitch Profile Design}

The first order assessment that helps bound the trade space comes from a parametric study of the performance of the varied pitch profiles on representative wind types. Since February produces the largest tailwinds and October the largest headwinds the average wind from each month was selected to represent Categories A and B respectively.

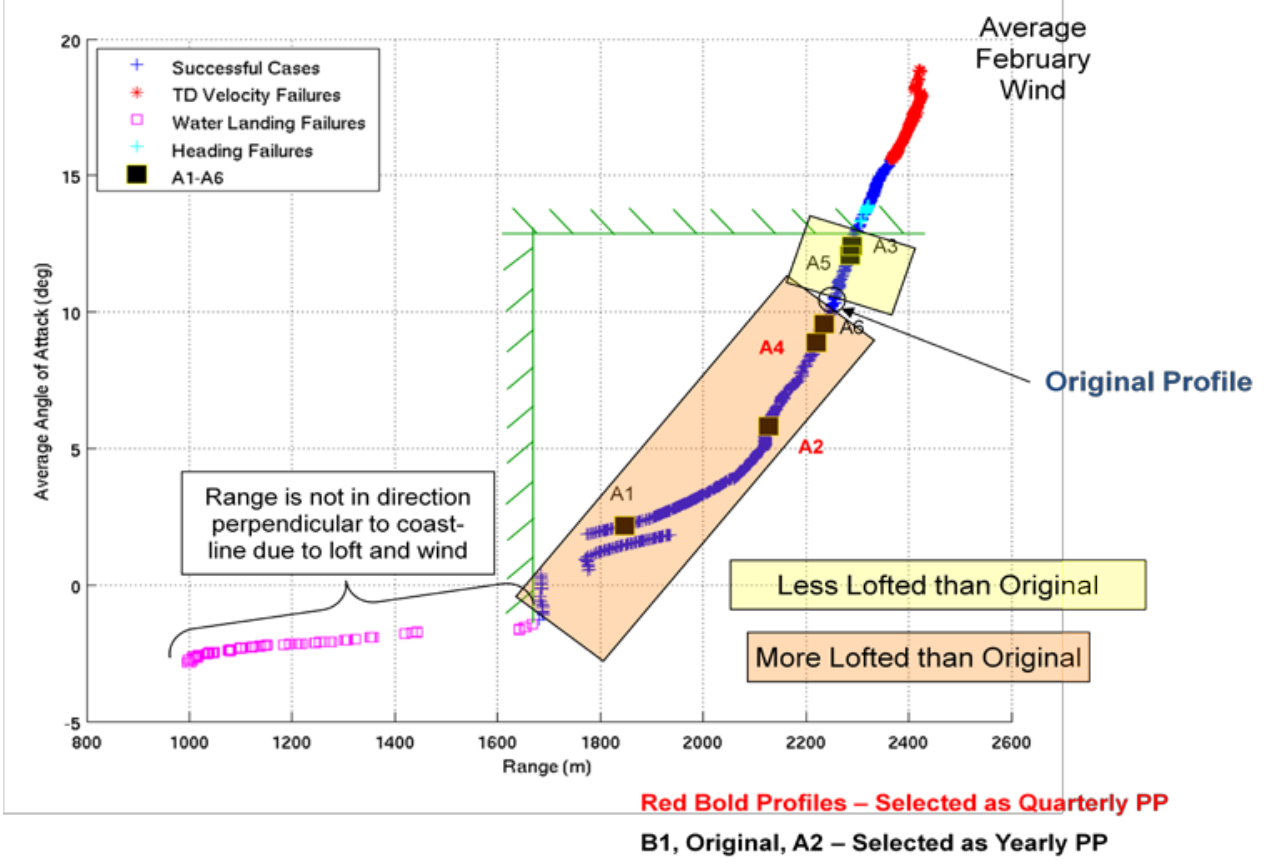

Figure 2. Category A (favorable headwinds) pitch profile design space

American Institute of Aeronautics and Astronautics 
Figures 2 and 4 are reproductions of the cartoon in Figure 1, but with actual trajectory data. Figure 2 shows the performance of a Category A wind with varying degrees of pitch. Each point on the curves in these figures comes from a unique profile designed as a table of three pitch angles that are time interpolated. The range was recorded at touchdown and the average angle of attack was calculated solely from the initial pitch over maneuver portion of the trajectory, around the time of the abort motor firing. Due to the favorable out to sea direction of the wind it was necessary to loft the wind with a negative angle of attack in order to get waterline failures. The increased loft of these trajectories prevented the vehicle from progressing directly to the water. As a result, the calculated range is not in the direction perpendicular to the coastline and the first water failure occurs at a range larger than the shortest distance from the pad to the coast on a line directly perpendicular to the coast.

From this data, a number of pitch profiles were evaluated as possible candidates for days with tailwinds. In this process, both depressed and lofted profiles were selected for the more detailed Monte Carlo analysis. "More lofted" and "less lofted" is defined relative to the original pitch profile that existed at Orion's Preliminary Design Review (PDR). Figure 3 displays the trends in performance metrics for some of the profiles considered. The competing constraints are immediately identifiable. In fact, profiles that result in land landings (waterline failures) result in trajectories that perform better in every other chute performance critical metrics including dynamic pressure at main line stretch and deviation from trim. If one profile was selected for all winds a compromise pitch profile that eliminated all touchdown velocity failures and minimized the waterline failures would have to be selected.

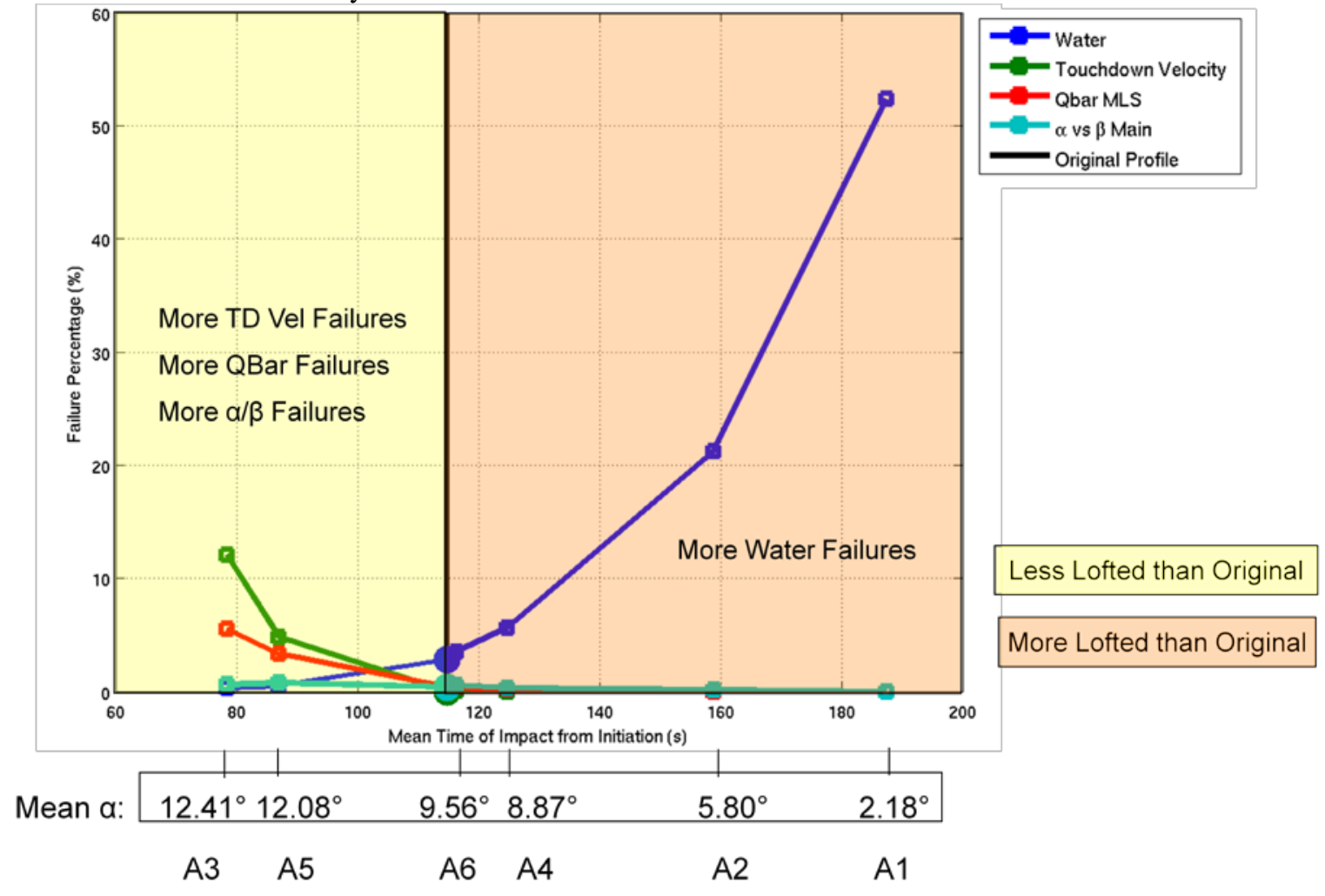

Figure 3. Degree of loft trade space; Failure percentage vs. trajectory time

The waterline failures noted in Figure 3 are all headwind cases in the broadest sense. For a tailwind, where the vehicle is already being pushed out into the ocean, a lofted trajectory is logical. Trajectories such as A2 and A4 that produce average angle of attacks less than 10 degrees are better suited for this categorization where a land landing is improbable especially if the chute deploy state is already over the water.

With a headwind, it takes a more depressed trajectory to avoid a land landing. Therefore, the first range where this occurs is approximately equal to the shortest range to the coastline $(1060 \mathrm{~m})$ as shown in Fig. 4. 


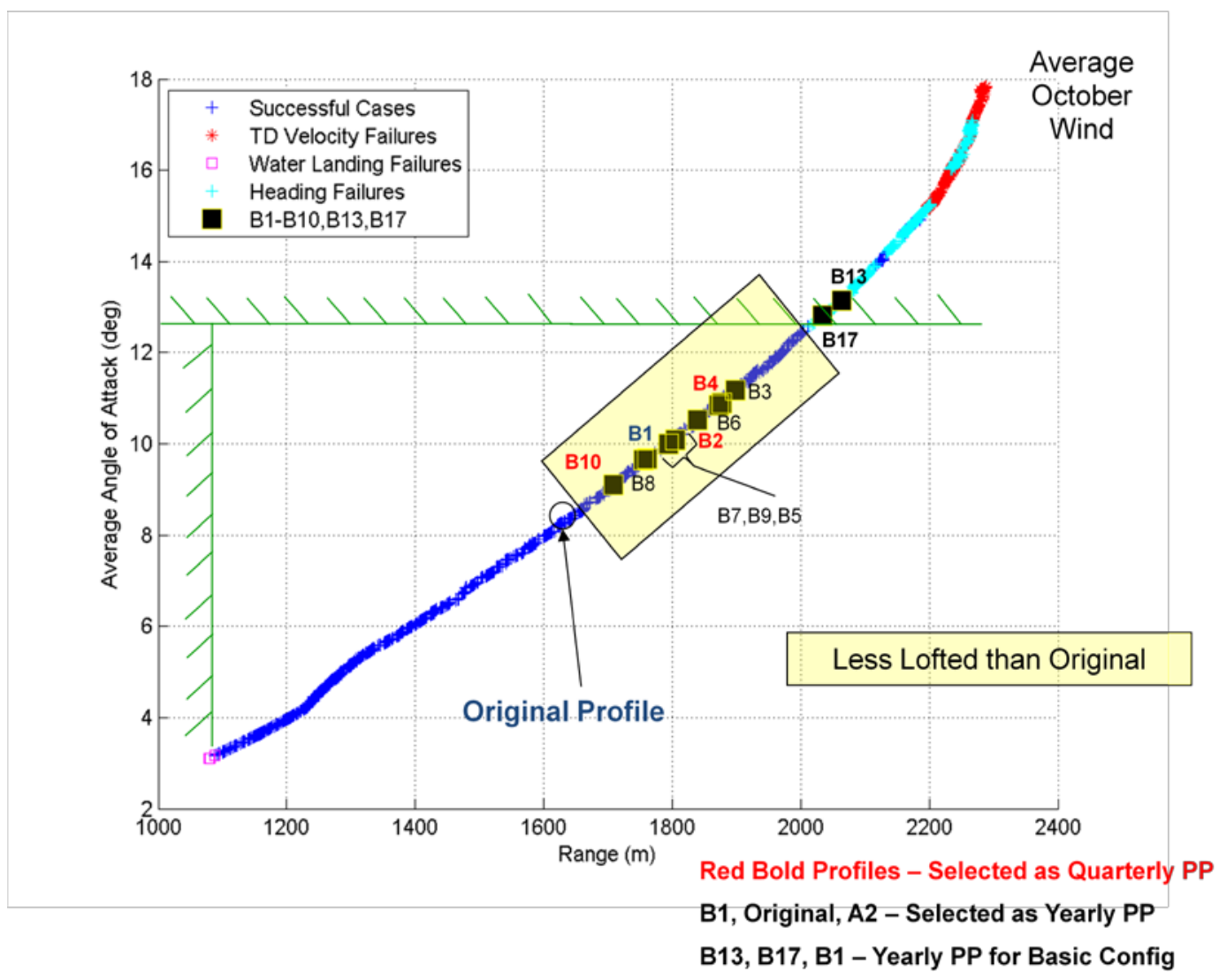

Figure 4. Category B (unfavorable tailwinds) pitch profile design space

While it appears more lofted trajectories exist within the acceptable bounds in Fig. 4 (to the left of the original profile), only less lofted profiles were evaluated. Nominal runs are useful in determining trends but an unknown amount of margin must be applied to the constraints in order to select a profile for a full Monte Carlo so that none of the cases violate the metrics. Therefore, the ideal headwind profile lays somewhere in the yellow box in Fig. 4, closer to the horizontal constraint. The number of cases between the constraint lines in Fig. 4 is much smaller than the number of cases in Fig. 2. The difficulty of determining the ideal profile is highlighted by the number of profiles evaluated for detailed Monte Carlo analysis as shown along the curve in Fig. 4.

\section{B. Wind Categorization by Season}

While at any time only three profiles are made available for selection, there could be a different set of profiles at different times of the year. To explore this idea, two types of categorization were considered: four sets of seasonal profiles versus one set for all seasons. These categorizations, referred to as the quarterly and yearly selections respectively, are based on mean wind values. The mean winds are placed in the different buckets (headwind, tailwind and compromise) by analyzing their value compared to a multiplier on the standard deviation from 0 (neutral wind). The mean wind values are calculated from winds that are positive blowing towards the North-East. In other words, tailwinds produce positive wind values, while headwinds produce negative winds. Each case average value is based on the latter half of the entire trajectory.

Figures 5-7 demonstrate the profile selection process for the different proposed configurations. These particular figures outline the procedure for the $4^{\text {th }}$ quarter winds as these months produce the largest headwinds and are therefore the most difficult to categorize for optimum success. The same process was repeated for all the other seasons. Figure 5 demonstrates the result of applying a heavily pitched profile (B4) to 2000 cases from this quarter. 


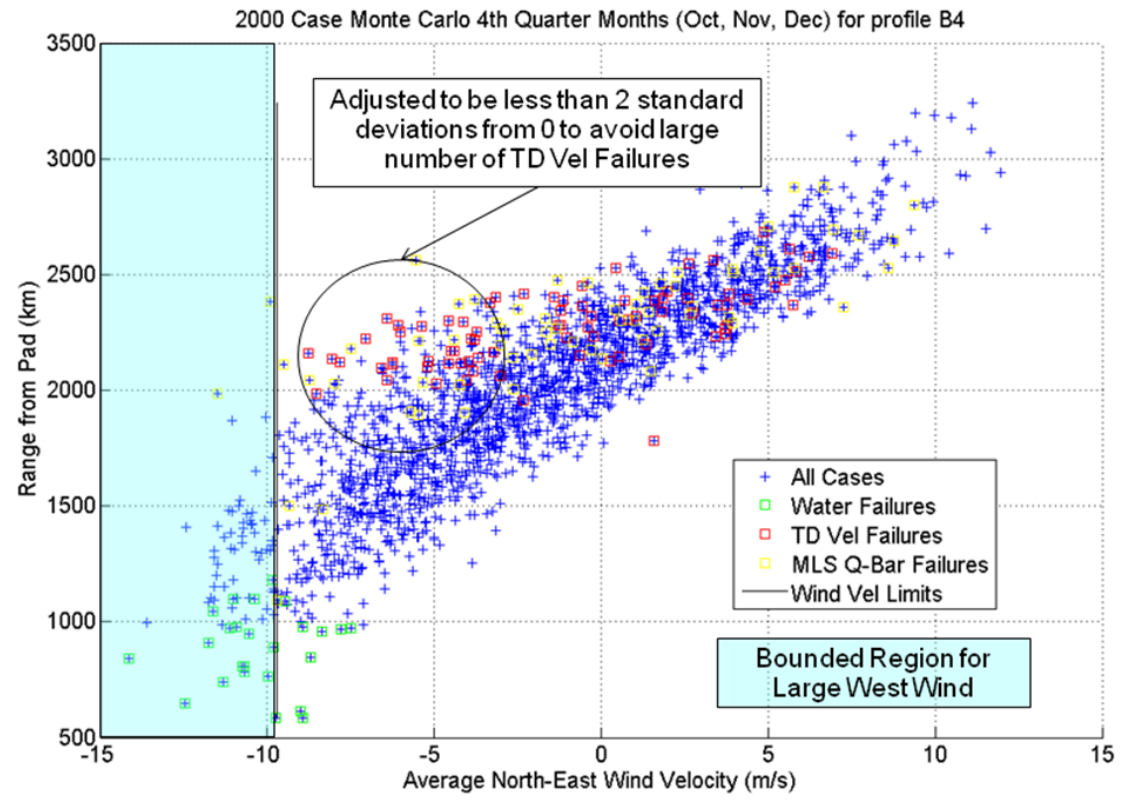

Figure 5. Headwind tailored profile assessment

There exists a general linear trend between average wind velocity and range from the pad, but this is clearly not a perfect trend. Many cases, in spite of the profile and large average headwind, tend to gather more range than others. Many of these cases, as shown by the circle in Fig. 5, exceed the touchdown velocity metric, which as mentioned previously, is a more critical failure than missing the water due to probability of loss-of-crew. This particular profile can only be applied to wind cases larger than 2 standard deviations less than 0 . It is really tailored for those strong October winds that will only succeed in reaching the water with guidance commands that produce an average angle of attack of $11^{\circ}$.

With the headwinds being limited to 2 standard deviations or lower from 0 , the compromise winds, from 2 standard deviations to 0, could actually be labeled as small to medium headwinds. This means that generally speaking, with even a small tailwind (the final category), the profile can be lofted. Figure 6 demonstrates that a slightly more aggressive profile than the original was selected for the compromise winds.

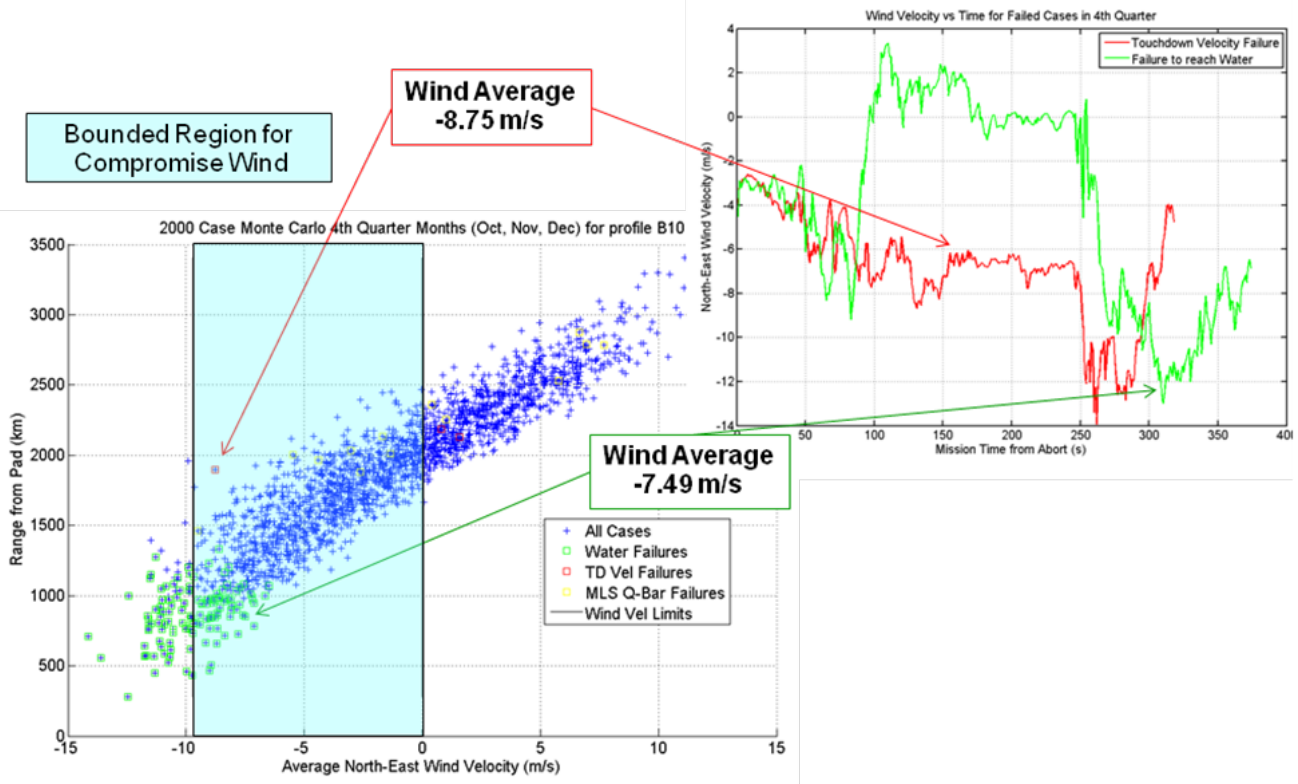

Figure 6. Compromise wind tailored profile trade

American Institute of Aeronautics and Astronautics 
The two cases highlighted in Fig. 6 show how other Monte Carlo dispersions impact the outcome. In fact, the case that was a touchdown failure is worse average headwind than the case that doesn't reach the water. If wind was the only factor, pitch profile design would be much simpler. In this case, dispersions on the launch abort motors had more of an effect than the winds.

The final category of winds is the tailwinds. Here, all of the winds have positive values and a lofted profile is successful in getting all of these cases to the water.

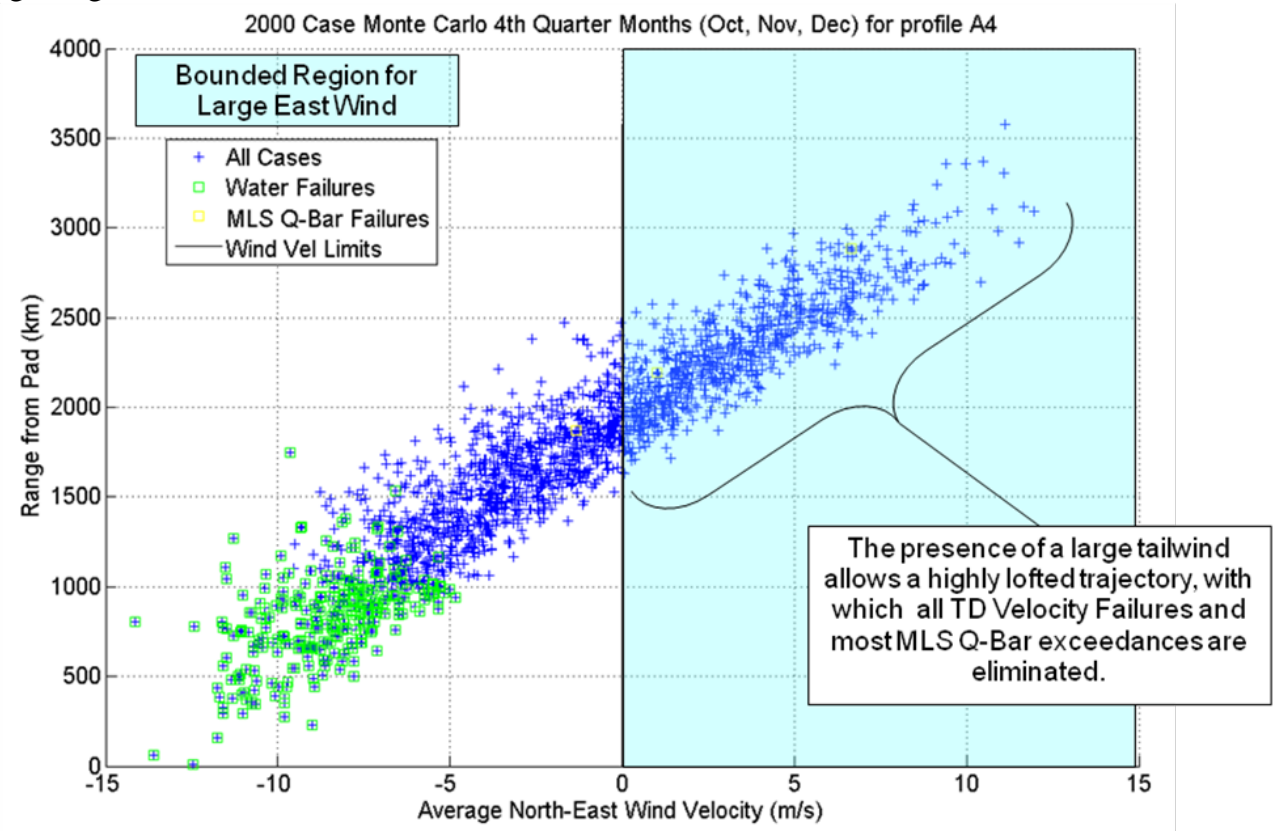

Figure 7. Tailwind tailored profile trade

While the plot in Fig. 7 demonstrates that the October-December quarter has more headwinds (values less than zero) than tailwinds, many tailwind cases remain. The use of quarterly designed winds loses its appeal when it is shown that every quarter and every month exhibits a large variation of winds. This is exhibited in Fig. 8 which compares all of the GRAM 2007 October (traditionally viewed as the headwind month) to February (traditionally viewed as the tailwind month) winds with a co-plot of the monthly means. The October mean is the lowest and the February mean is one of the highest, but both months have winds all across the board. Note: GRAM 2007 is the official atmosphere and winds model for the Orion Project and the Constellation Program.

\section{October Winds with Monthly Means}

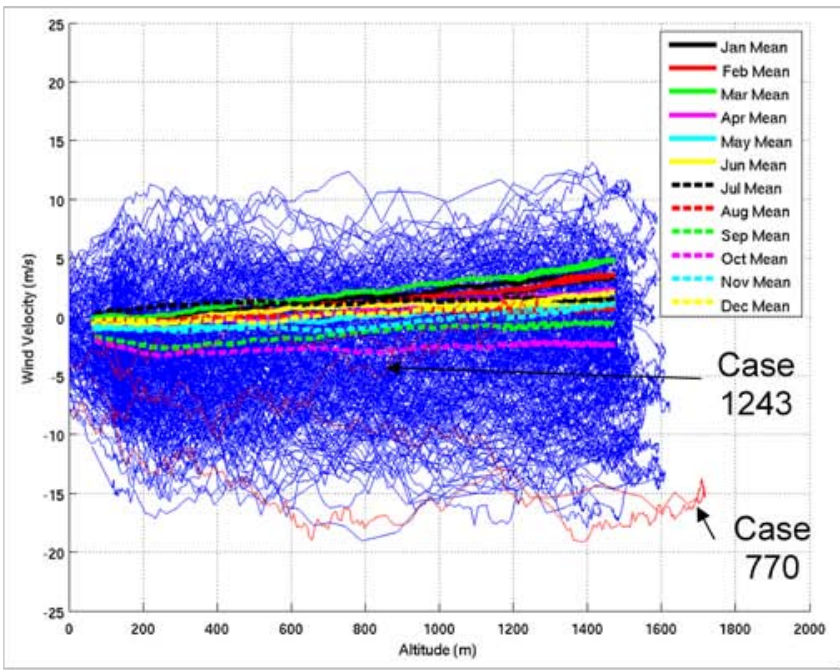

\section{February Winds with Monthly Means}

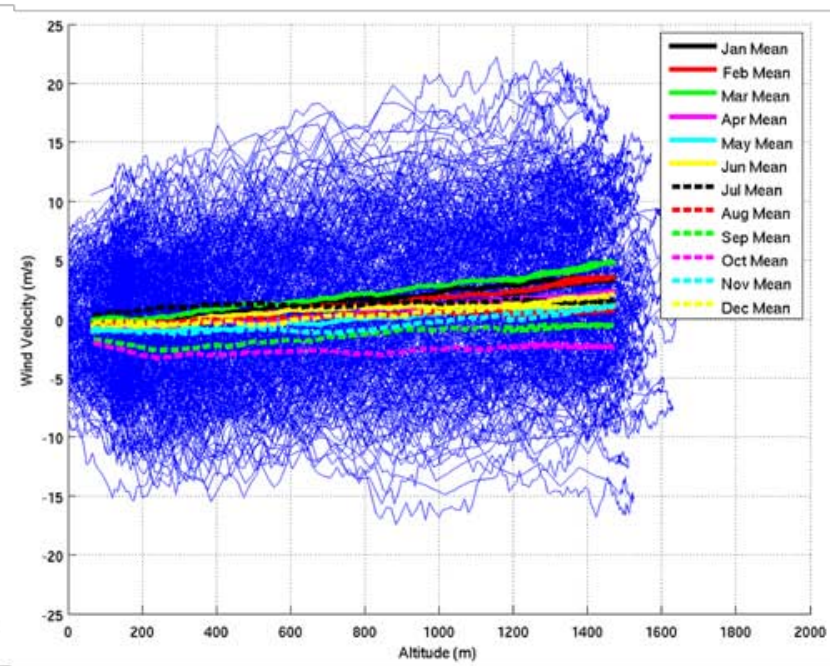

Figure 8. Comparing October and February winds with monthly means 


\section{Water Failure Reduction with Profile Selection}

Once the profiles are selected, the resultant performance can be assessed. The bar chart in Fig. 9 shows the change in the target metrics based on the profile selections for an 8000 case Monte Carlo with 2000 cases randomly selected from each quarter throughout an entire year.

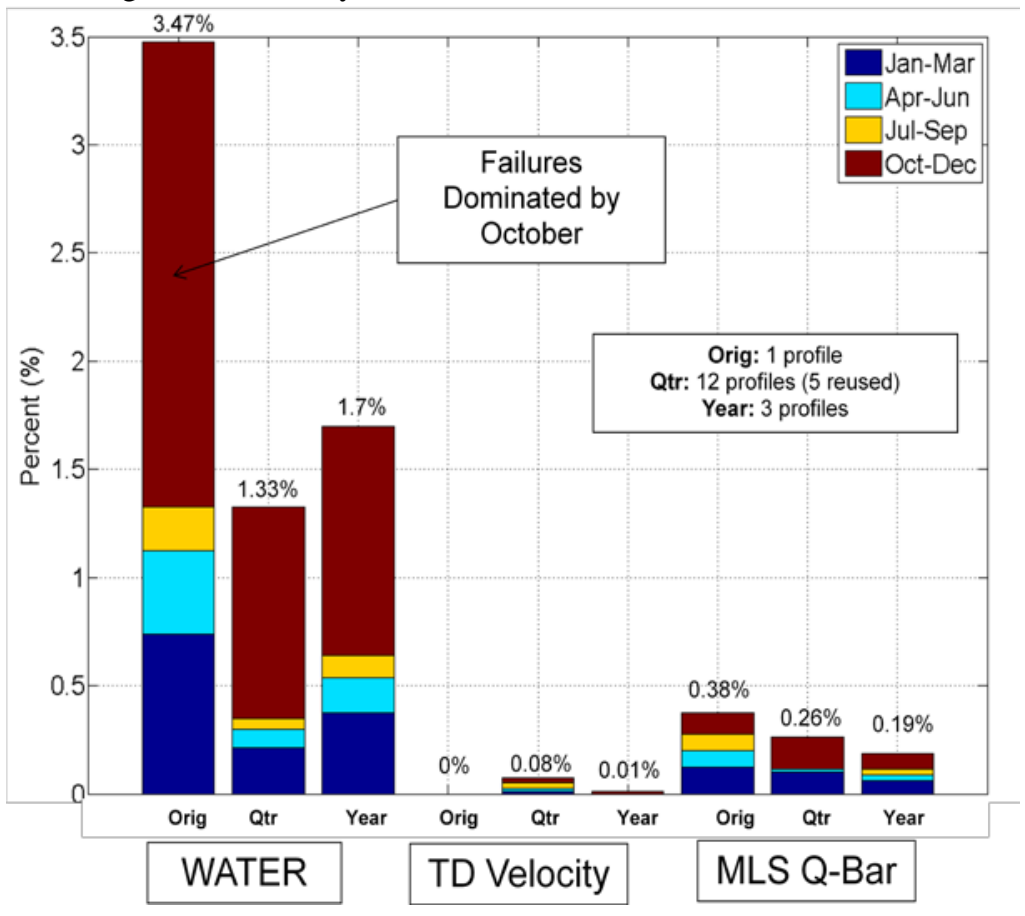

Figure 9. Failure reduction for $\mathbf{8 0 0 0}$ case Monte Carlo with varying types of profile selection

The size of the bar indicates the total number of failures, while the different colors show the failure breakdown by season. In addition to the waterline and touchdown velocity failure metrics, the dynamic pressure limit failure metric for the main chutes was added as a key performance driver. The main observation to draw from Fig. 9 is the large decrease in waterline failures. In both the quarterly and yearly profile selection modes the number of failures is cut approximately in half with a slight increase in touchdown velocity failures and a slight decrease in dynamic pressure failures at main line stretch. The quarterly based profiles are more effective than the full year profiles in driving down waterline failures but at the expense of increased touchdown velocity and main line stretch dynamic pressure failures.

It is important to note that it is by design that the touchdown velocity metric remains close to zero as this metric is vital for crew survival. In fact, the profiles were designed (with fewer Monte Carlo runs) to keep this metric at zero. However, since the pitch profiles are aggressive, there is an increased risk of a chute failure. If all failures were equal, which they are not, the overall minimum failure percentage would contain a higher percentage of touchdown velocity failures along with a lower percentage of waterline failures. But this is not a compromise the Orion community is prepared to make.

\section{Wind Placard Changes with Profile Selection}

While the final percentage of failures using winds on the day of launch to choose pitch profiles may be acceptable to project management. It is more likely that Orion will placard out the winds that are proving the most problematic in order to reduce the overall probability of loss of crew during an abort even lower. Thus, an initial process for determining these placards was investigated.

In order to determine wind placards based on wind conditions that can be extremely unpredictable and variable, it is necessary to determine what types of winds will cause failures, in this analysis those that result in land impact. The wind categorization developed for the placards is based on average wind value and the average azimuth direction of a particular wind of the day. Of course, winds constantly change direction and magnitude and depend on altitude. While an average wind might give an idea of the general course of a particular wind it doesn't account for gusts or the direction of that particular gust or the altitude that is most problematic. Accounting for gusts or where within the 0 to $4000 \mathrm{~m}$ altitude band the wind is strongest, does not improve the wind placard categorization 
significantly. Thus, it should not be too surprising that there is a wide range of azimuths (within a region near the headwind direction of 57deg) that produce unacceptable water failures. Figure 10 below shows different percent failure levels for given azimuth wedges of $20^{\circ}$ ranging from $95 \%$ to $100 \%$ for the original pitch profile. Figure 11 shows the improvement in percentage levels for the select 3 pitch profiles. For example, if the wind placard is set at 95\%, the azimuth coverage improves from $300 \mathrm{deg}$ to $320 \mathrm{deg}$ (of a total $360 \mathrm{deg}$ ) and the restricted 40 degree range has a $1-3 \mathrm{~m} / \mathrm{s}$ higher average wind speed limit.
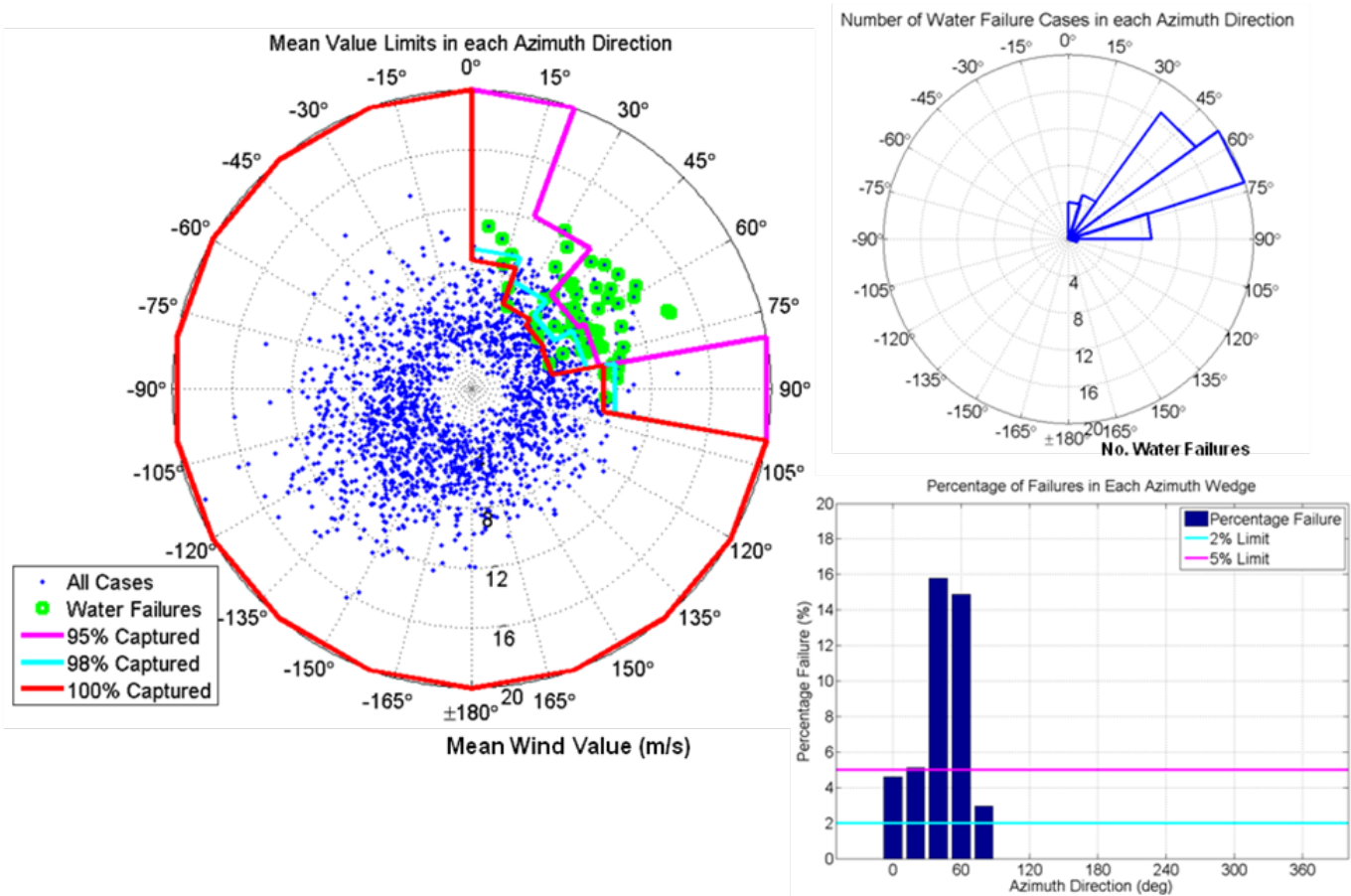

Figure 10. Original Profile water failures; percentages and tallies calculated in $20^{\circ}$ intervals
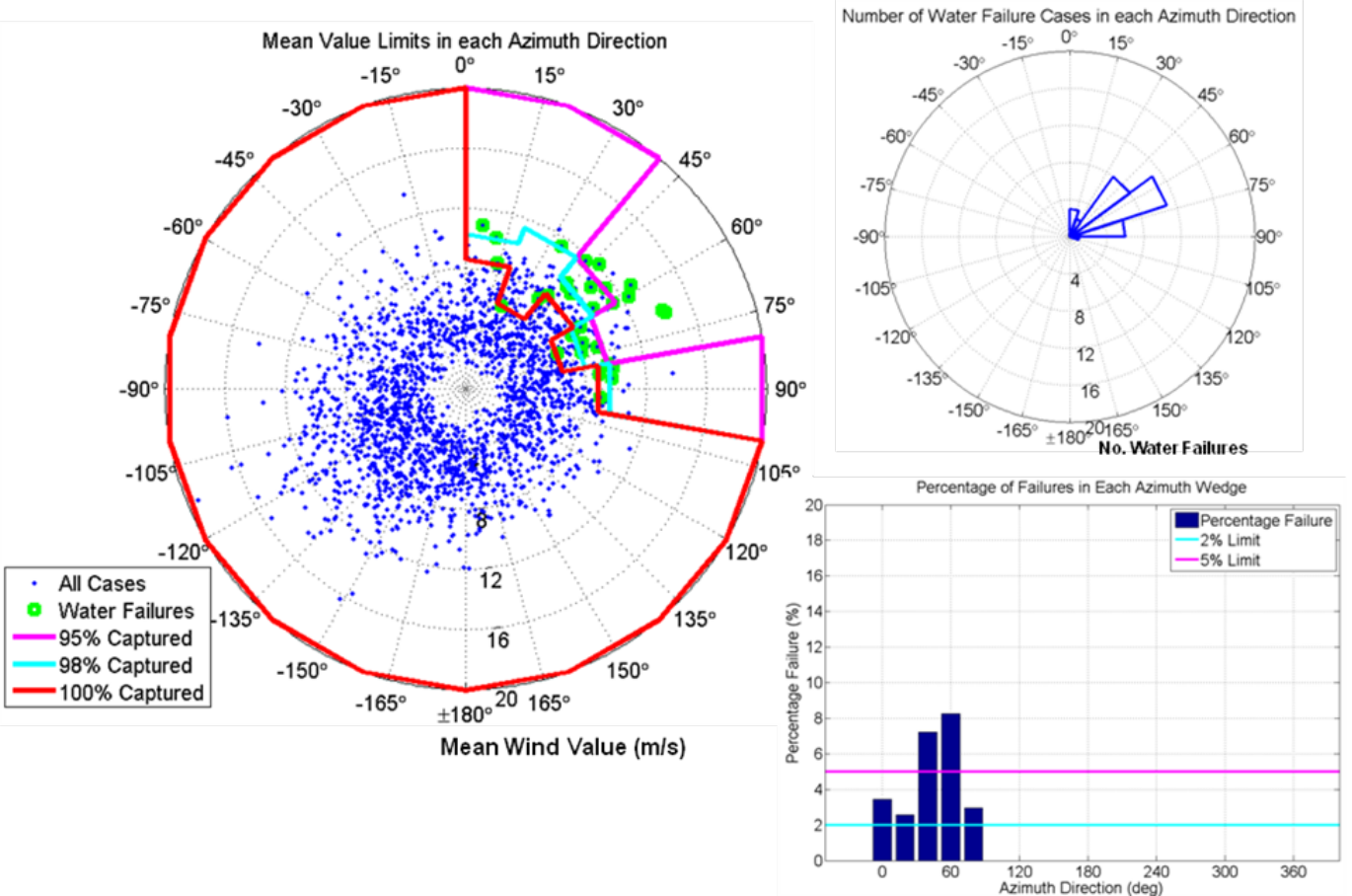

Figure 11. Select 3 Profile water failures; percentages and tallies calculated in $20^{\circ}$ intervals

American Institute of Aeronautics and Astronautics 


\section{Straight to Main Chute Architecture Results}

\section{A. Dynamic Pressure Designed Pitch Profiles}

In the drogue to main chute architecture study, the pitch profile table contained only three values from which the whole profile was interpolated. In the new control algorithm formulation used in the straight to main design, any time dependent $10 \mathrm{~Hz}$ profile desired can be generated. In addition, instead of setting a pitch Euler angle target, the control is (flight path angle rate), roughly equivalent to pitch rate for pad abort cases. Figure 12 shows the final profile parameterization designed for this analysis.

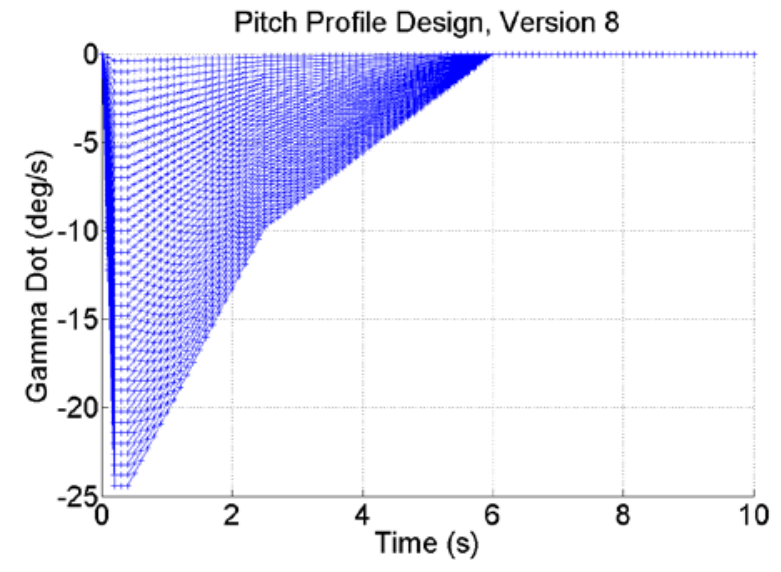

Figure 12. Control parameterization of equivalent to pitch rate

A desired average angle of attack can be achieved by commanding a short duration, high impulse profile, but this tends to induce tumbling due to a large increase in aero torques during the abort motor phase (at $t=0-2$ seconds). This particular parameterization avoids this problem by spreading the pitch rate across the first six seconds.

In the straight to mains architecture formulation the timeline is shifted. Eliminating the drogues adds time to the trajectory but additional delays in Forward Bay Cover (FBC) jettison remove time. In this shifted sequence, dynamic pressure at main line stretch becomes a larger concern. At the beginning of the straight to mains analysis the main line stretch dynamic pressure limit was 85 psf. Based on the drogue to main chute analysis, a 20-25 psf margin should be added to the nominal to encompass all dynamic pressure dispersions in the Monte Carlo. In order to design this margin into the profiles from the start, the average angle of attack vs. range plot in Fig. 13 includes dynamic pressure contours for the main chutes in psf units. Note that Fig. 13 uses the parameterized pitch profiles shown in Fig. 12.

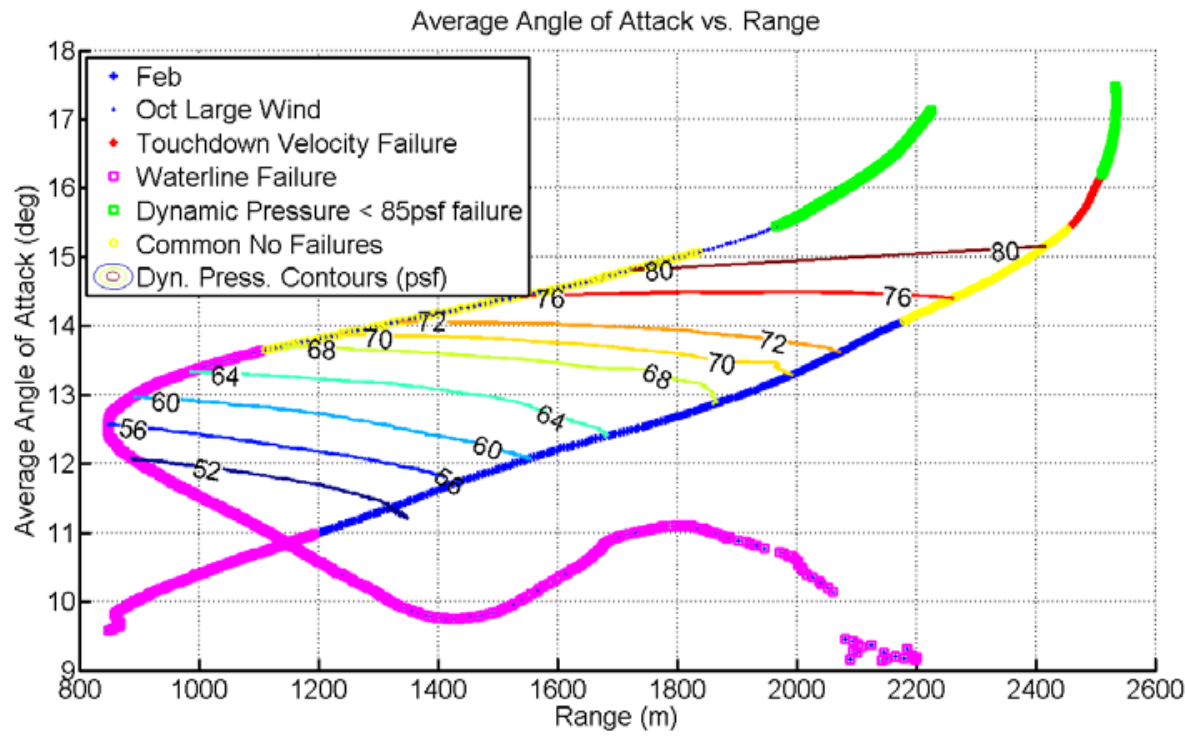

Figure 13. Relationship of pitch profile design to range with failures

American Institute of Aeronautics and Astronautics 
Pitch profile parameterizations that produced an undispersed dynamic pressure of around 60 psf appear to be ideal candidates to meet the 85 psf dispersed limit. Figure 14 below shows how much ride time (in terms of altitude at main inflation) is available given for different dynamic pressure contours and for the different pitch profiles shown in Fig. 12. As expected, the February (Category A) wind has reduced altitude available compared to the October wind (Category B).

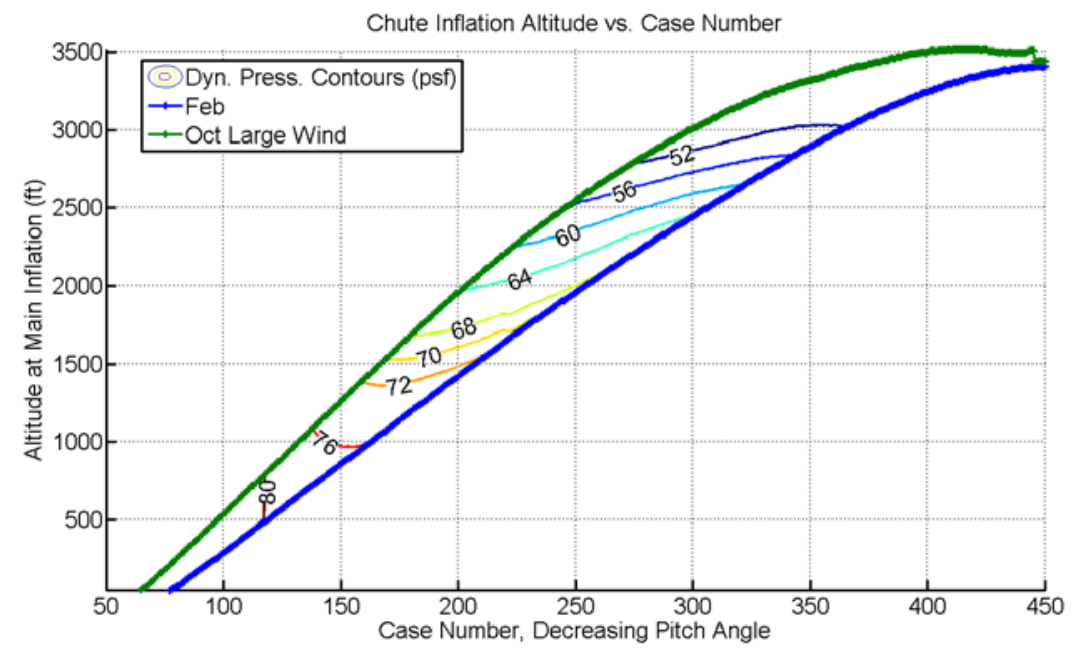

Figure 14. Main inflation altitude increase with loft

Figures 13 and 14 demonstrate the trade space well and are very useful in the evolving design. With this parametric, profiles could be selected even when the dynamic pressure limit is changed by adjusting the nominal accordingly.

\section{B. Land Landing Failure Reduction with Profile Selection}

During the course of this study the dynamic pressure limit for main line stretch jumped from 85 to $120 \mathrm{psf}$. A favorable change, it has a positive impact on the degree in which waterline failures can be mitigated successfully. Profiles were selected with both the 85 psf and 120 psf limits in mind but only the 120 psf results are presented here. For Category A profiles starting at about the 70 psf contour (approximately case number 225 in Fig. 14), tumbles and touchdown altitude rate failures begin to appear in the Monte Carlo. If the estimated 20-25 psf margin added to the nominal bounds the worst Monte Carlo dynamic pressure case, the worst case would still below 100 psf. The change in margin made the touchdown velocity limit and tumbling metric the driving constraints.

In contrast to the drogue to main design where standard deviations were applied to North-West average wind values, the selection criteria was based solely on average wind magnitude and azimuth. As in the drogue to main study, the headwinds comprise the smallest category of cases because they are the rarest. In this instance, the headwinds are said to be those winds in the 25 to 80 degree azimuth band with an average wind speed greater than $7.5 \mathrm{mph}$. The average wind profile is applied to the rest of the cases between - 30 and 135 degrees (a 165 degree swath). The tailwind cases include the remaining 195 degrees of wind azimuth and may have any wind magnitude.

For the straight to main cases, 2000 case Monte Carlos are run. Table 1 shows the change in the performance metrics with the select 3 profile compared to the original. Regardless of loft, one case always tumbled and then subsequently violated the touchdown metric. Figure 15 demonstrates these results graphically.

Table 1. Performance increase due to select 3 algorithm

\begin{tabular}{|c|c|c|c|c|c|}
\hline & $\begin{array}{l}\text { Original } \\
\text { Profile }\end{array}$ & $\begin{array}{l}\text { Tailwind } \\
\text { Profile }\end{array}$ & $\begin{array}{l}\text { Average Wind } \\
\text { Profile }\end{array}$ & $\begin{array}{l}\text { Headwind } \\
\text { Profile }\end{array}$ & $\begin{array}{c}\text { Combined from } 3 \\
\text { Select Profiles }\end{array}$ \\
\hline Tumbling & $1(0.1 \%)$ & 1 (0.1\%) & $1(0.1 \%)$ & $11(0.5 \%)$ & $1(0.1 \%)$ \\
\hline Main Line Str.: Q-Bar & $0(0.0 \%)$ & $0(0.0 \%)$ & $0(0.0 \%)$ & $1(0.1 \%)$ & $0(0.0 \%)$ \\
\hline Touchdown: Alt. Rate & $1(0.1 \%)$ & 1 (0.1\%) & $1(0.1 \%)$ & $13(0.7 \%)$ & $1(0.1 \%)$ \\
\hline $\begin{array}{c}\text { Touchdown: } 10 \mathrm{ft} \\
\text { Waterline }\end{array}$ & $52(2.6 \%)$ & $148(7.4 \%)$ & $52(2.6 \%)$ & $14(0.7 \%)$ & $24(1.2 \%)$ \\
\hline
\end{tabular}



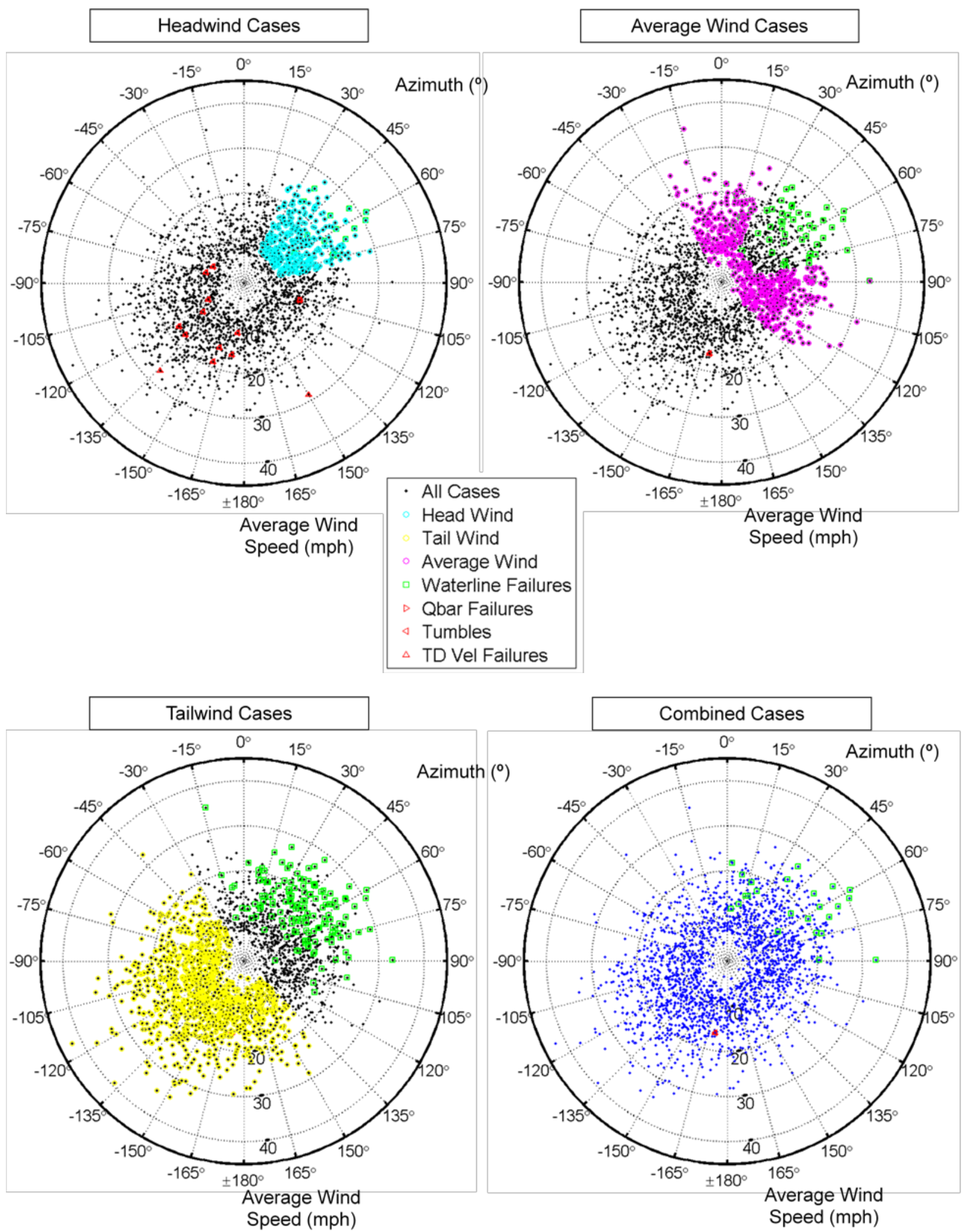

Figure 15. Pitch profile selection and waterline failure metric reduction

American Institute of Aeronautics and Astronautics 
In Table 1, the nominal performance for straight to mains is very comparable to the drogue to main chute architecture. With fewer initial waterline failures (2.6\% compared to $3.5 \%$ ) fewer failures also exist in the combined set. In this case, only 24 out 2000 cases fail to reach the water or 1.2\% (compared to $1.7 \%$ for drogues to mains).

\section{2hr Shuttle Wind Pairs Results}

The analysis completed so far assumes perfect wind knowledge which is in fact impossible. In order to demonstrate how inaccuracies in wind knowledge affects the performance of the select 3 algorithm a 567 case set of time delayed wind pairs were incorporated into the study. These wind pairs are used by the Space Shuttle Program for its day-of-launch analysis activities. The pitch profile selection is made based on data from the first wind, and then the actual real time wind data from the second is applied in the trajectory simulation.

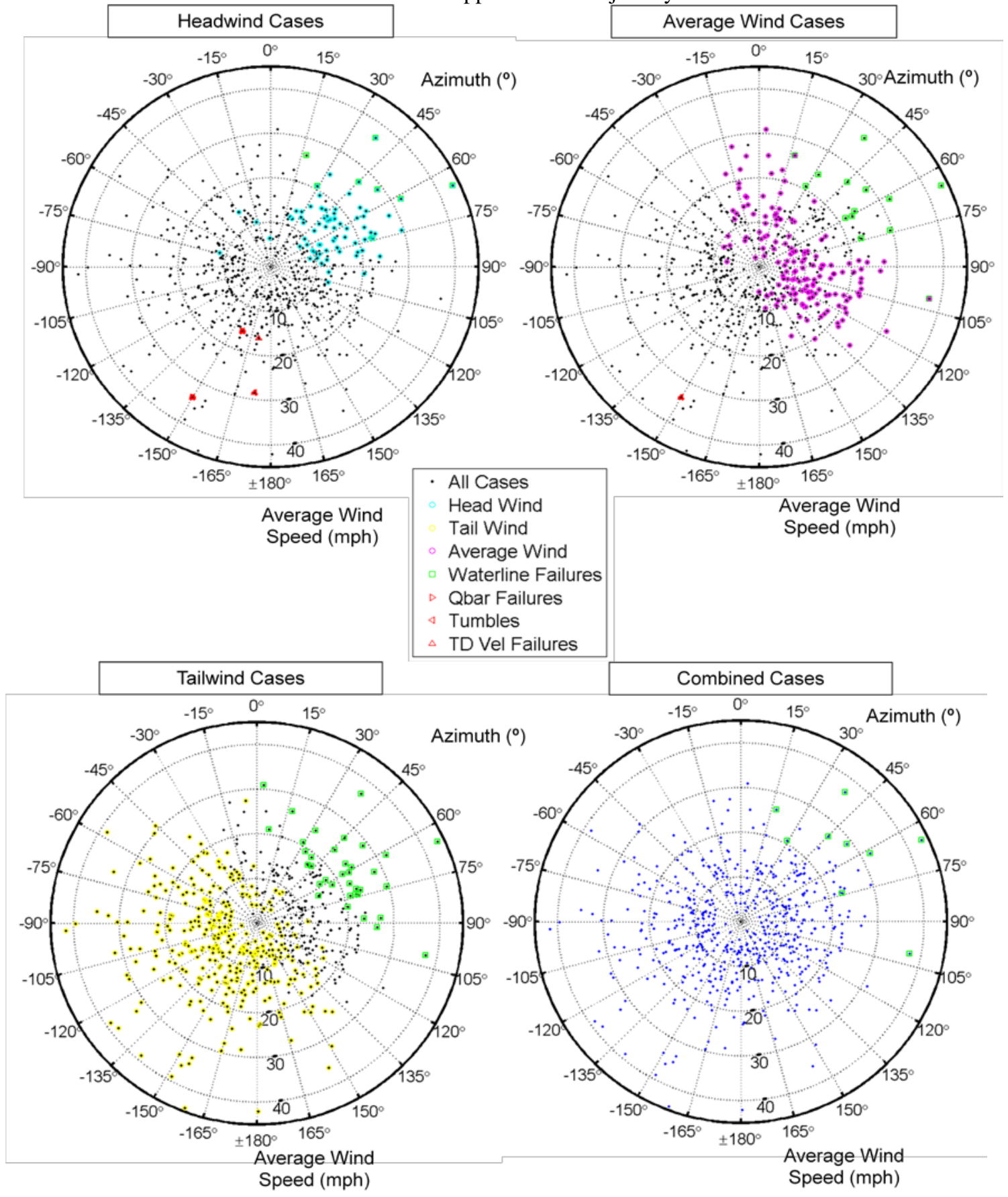

Figure 16. Affect of measurement delay on algorithm performance

12

American Institute of Aeronautics and Astronautics 
Figure 16 shows the resultant performance. The same pitch profiles used in the 2000 case Monte Carlo are again selected here using the same azimuth and wind speed criteria. During the 2 hours that elapses in-between the measurement and the actual flight, the winds do change. Figure 16 shows that many cases selected for a certain profile are no longer in the correct category. In this example, the wind variance appears to make little difference; when categorized based on the real-time wind, the reduced waterline failure percentage is the same. In addition, the results are consistent with the GRAM 2007 based 2000 case Monte Carlo. The 2000 set reduced the waterline failure percentage from $2.6 \%$ to $1.2 \%$ with no other failure metric increases and the shuttle wind pairs demonstrate a reduction from $2.5 \%$ to $1.6 \%$, also without other metric increases.

\section{Conclusions}

This analysis has demonstrated the benefits of incorporating an array of profiles based on day-of-launch wind data into the pad abort guidance design. The proposed baseline array size is 3, designed for the entire year instead of by season or by month. In general, selecting from multiple open loop pitch profiles instead of one reduces the number of waterline failures significantly. The overall level of reduction depends heavily on the pad abort sequence and vehicle design. For the Orion LAV straight to mains design, selecting from 3 profiles eliminates approximately half of the waterline failures.

\section{References}

1 “Guidance Navigation \& Control Data Book,” LM CEV-T-078, 2008. 